\title{
A truly hybrid approach to perventricular closure of multiple muscular ventricular septal defects
}

\author{
Karim A. Diab, MD, ${ }^{a}$ Ziyad M. Hijazi, MD, ${ }^{a}$ Qi-Ling Cao, MD, a and Emile A. Bacha, MD, ${ }^{\text {b }}$ Chicago, III
}

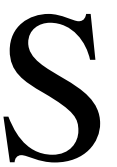

urgical closure of muscular ventricular septal defects (VSDs) remains a challenging problem. Percutaneous device closure in infants remains challenging. ${ }^{1}$ Perventricular device closure on the beating heart has been described previously. ${ }^{2}$ Some VSDs cannot be crossed through the right ventricular (RV) free wall puncture. We report the case of an infant with two significant VSDs who underwent successful perventricular device closure with a truly hybrid technique.

\section{Clinical Summary}

A 4-month-old, 5.7-kg infant was referred to our center for perventricular closure of 2 VSDs. The procedure was performed in the catheterization suite as part of a live course to demonstrate the technique of hybrid intervention. Transesophageal echocardiography (TEE) revealed the presence of two nonrestrictive VSDs (9-mm apical and 7-mm anterior defects; Figure E1). The right femoral artery was accessed percutaneously, and left ventricular (LV) angiography demonstrated the defects (Figure 1). A 3-cm incision was made over the typhoid process, and the RV free wall was exposed. With a previously described technique, ${ }^{2}$ the apical VSD was easily crossed with a wire. Because of crowding of the RV apex with muscle bundles, we could not expand the RV disk of the 10-mm Amplatzer MVSD Occluder (AGA Medical Corp, Golden Valley, Minn) that had been chosen initially. The device was therefore recaptured in the sheath, and an Amplatzer Duct Occluder (AGA Medical) was used instead. The duct occluder has only one disk and a stalk, similar to a mushroom shape. This VSD was successfully closed. TEE revealed significant shunt through the anterior VSD. Multiple attempts at crossing this VSD through multiple puncture sites in the RV free wall failed. A $4 \mathrm{~F}$ Judkins right coronary catheter was therefore advanced percutaneously under fluoroscopic guidance through the femoral artery retrogradely into the LV. A 0.035 -inch glide wire was then manipulated

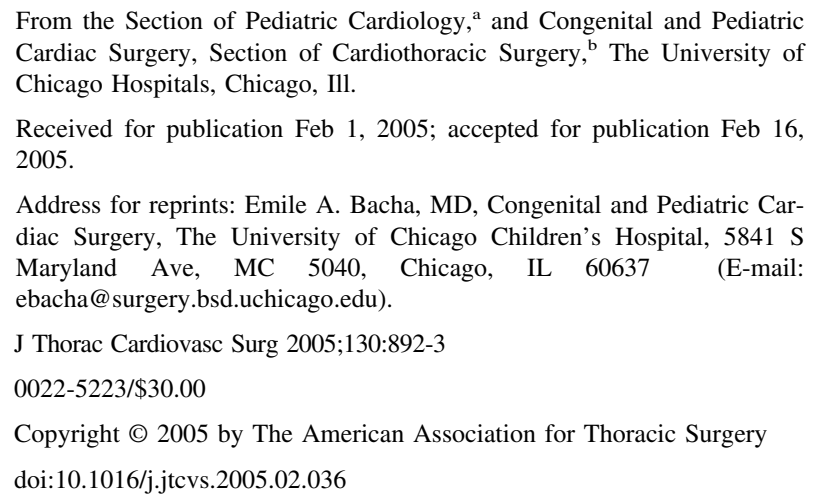

inside this catheter until it crossed the VSD into the pulmonary artery (PA). Through the RV free wall puncture, a wire and then a 7F short sheath were positioned in the main PA under fluoroscopy. A 4F multipurpose catheter with a $10-\mathrm{mm}$ gooseneck snare (ev3, Inc, Plymouth, Minn) was advanced inside this sheath. Under fluoroscopic guidance, the glide wire was snared and exteriorized out through the RV free wall, forming an arterial-RV free wall loop. Over this wire loop, the short $7 \mathrm{~F}$ sheath was directed toward the VSD into the LV cavity. The wire was removed, and the sheath position was confirmed by angiography and TEE. An 8-mm Amplatzer MVSD Occluder was then deployed with regular perventricular technique. TEE revealed complete closure of the second defect. The patient was discharged to home on postoperative day 3. Echocardiography at 3 months revealed complete closure of the two defects. The patient has been gaining weight normally and is not receiving any medications.

\section{Discussion}

This report describes the management of multiple VSDs in an infant with congestive heart failure. We had been performing perventricular device closures in the operating room under TEE guidance alone. Because of the extremely anterior location of one of the VSDs, we anticipated that fluoroscopy would be needed in this case. In fact, fluoroscopy proved to be essential in that we incorporated elements and steps typical of percutaneous device closure into our perventricular technique, resulting in a truly hybrid procedure. On the basis of this experience, we now prefer to perform perventricular closures in the catheterization laboratory if no other concomitant surgical repairs are needed. This allows us to use the full extent of percutaneous techniques, along with the advantages of performing direct cardiac access procedures. Some VSDs, in particular apical ones, are easier to cross from the left side, because there is often a single hole on the smooth, nontrabeculated left-sided ventricular septum. ${ }^{3}$ Placing a wire or catheter into the PA through RV free wall puncture is a routine step for heart surgeons and allows the creation of an arteriovenous loop. Once a connection between the RV free wall and the VSD is established, perventricular device placement is usually routine. Advantages relative to a purely percutaneous approach include preservation of venous access, because no large sheaths are used, and lessened chances of aortic or tricuspid valve injury or cardiac perforation. The anterior VSD could have easily been found and repaired with traditional surgery, but this would have required cardiopulmonary bypass and cardioplegic arrest. To our knowledge, this is the first such case in which percutaneous and perventricular approaches have been combined to close a muscular VSD, moving from collaboration of practitioners to integration of disparate techniques into a truly hybrid procedure. 

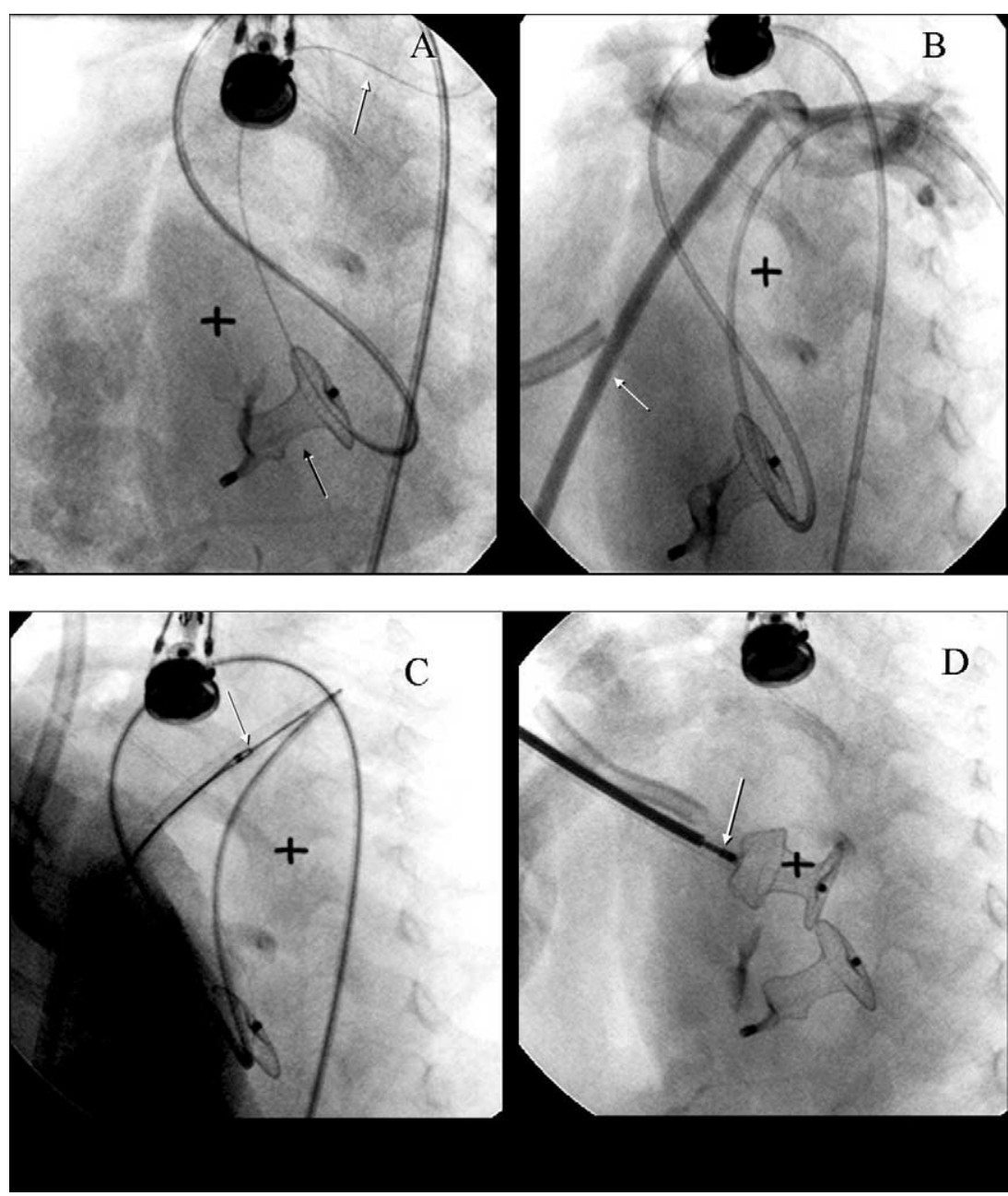

Figure 1. Cineangiographic images in the hepatoclavicular position demonstrating closure procedure. A, Cineangiographic image after Amplatzer Duct Occluder (black arrow) has been released and anterior muscular VSD has been crossed from the left side with wire positioned in the left PA (white arrow). B, Hand-injection angiogram in the main PA through a delivery sheath (short arrow) passed from the RV free wall to the main PA (long arrow showing a red rubber tourniquet used for the purse-string suture). C, Cineangiographic image during snaring of wire from the PA inside the delivery sheath (arrow). D, Cineangiographic image during deployment of the RV disk (arrow). The LV disk is already deployed. The device is inserted through the RV free wall.

\section{References}

1. Holzer R, Balzer D, Cao QL, Lock K, Hijazi ZM. Amplatzer Muscular Ventricular Septal Defect Investigators. Device closure of muscular ventricular septal defects using the Amplatzer muscular ventricular septal defect occluder: immediate and mid-term results of a U.S. registry. J Am Coll Cardiol. 2004;43:1257-63.

2. Bacha EA, Cao QL, Starr JP, Waight D, Ebeid MR, Hijazi ZM. Perventricular device closure of muscular ventricular septal defects on the beating heart: technique and results. J Thorac Cardiovasc Surg. 2003; $126: 1718-23$.

3. Tsang VT, Hsia TY, Yates RW, and Anderson RH. Surgical repair of supposedly multiple defects within the apical part of the muscular ventricular septum. Ann Thorac Surg. 2002;73:58-62. 

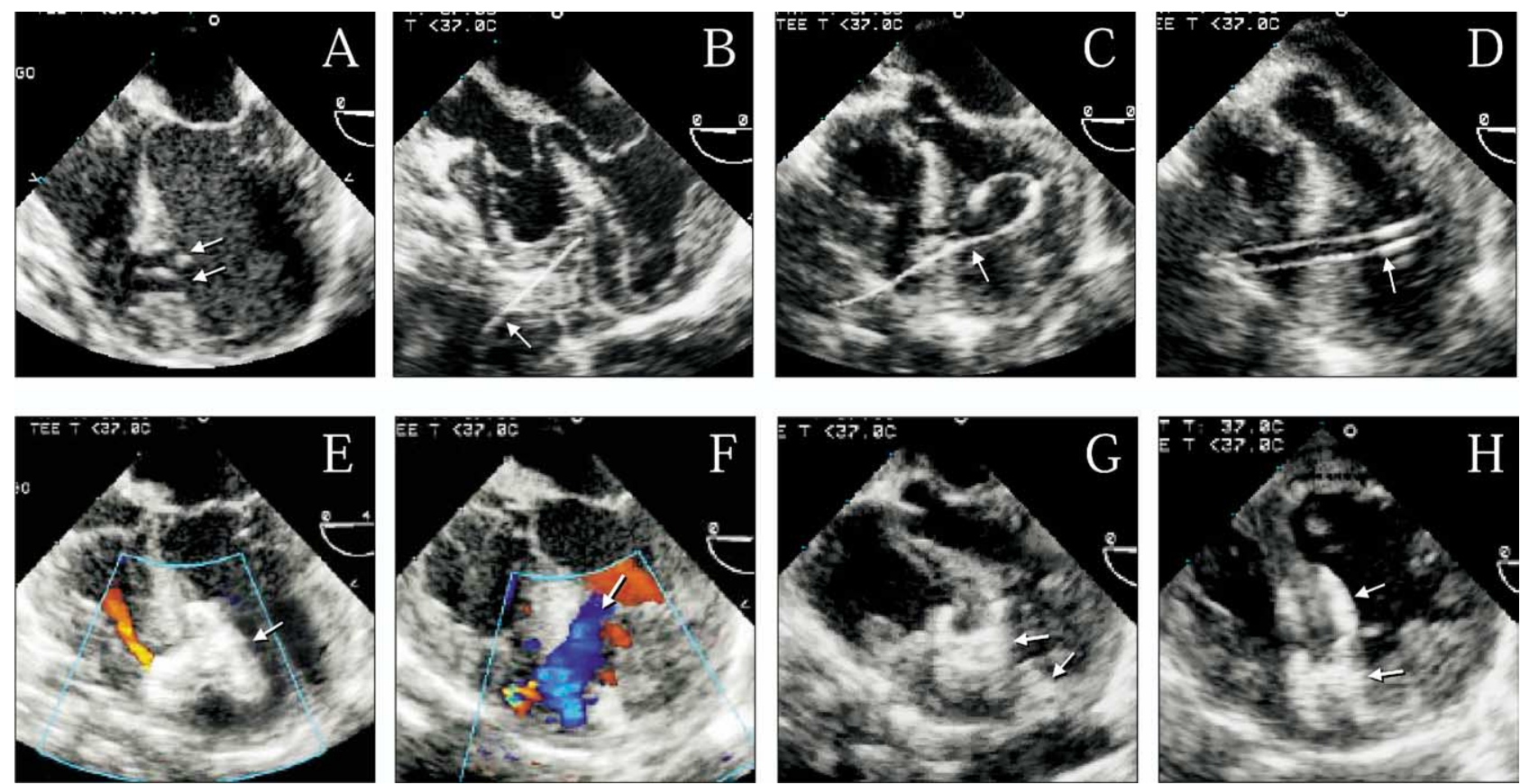

Figure E1. TEE images in four-chamber views (except $H$ ) demonstrating the closure procedure. A, Demonstrates two defects, near the apex and more midanterior (arrows). B, Puncture of the RV free wall with an 18-gauge needle (arrow). C and D, Passage of the guide wire (C) and delivery sheath (D) through the apical VSD to the LV cavity. E, Amplatzer Duct Occluder was released, demonstrating good position. F, Color Doppler demonstrates a large residual shunt from the anterior VSD. G and H, After the Amplatzer muscular VSD device has been released, both devices (arrows) are in good position. 